\title{
A Hipótese da Aparência Ecológica pode explicar a importância local de recursos vegetais na região do Parque Nacional de Sete Cidades, Piauí, Brasil?
}

\section{Derly Pereira Brasileiro ${ }^{1}, *$, Ezequiel da Costa Ferreira ${ }^{1}$, Suellen da Silva Santos ${ }^{1}$, Thamires Kelly Nunes Carvalho ${ }^{1}$, João Everthon da Silva Ribeiro ${ }^{3}$, Roseli Farias Melo de Barros $^{2}$, Camilla Marques de Lucena ${ }^{3}, * *$ e Reinaldo Farias Paiva de Lucena ${ }^{1,3, * * *}$}

\begin{abstract}
${ }^{1}$ Universidade Federal da Paraíba. Centro de Ciências Exatas e da Natureza. Programa de Pós-Graduação em Desenvolvimento e Meio Ambiente - PRODEMA. João Pessoa-PB, Brasil (CEP 58051-900).*E-mail: pfbrasileiro@yahoo.com.br.

${ }^{2}$ Universidade Federal do Piauí. Programa de Pós-Graduação em Desenvolvimento e Meio Ambiente - PRODEMA. Teresina-PI, Brasil (CEP 64049-550).

${ }^{3}$ Universidade Federal do Mato Grosso do Sul. Instituto de Biociências. Laboratório de Etnobotânica. Campo Grande-MS, Brasil (CEP 79070-900). **E-mail: camilla.lucena@ufms.br; ${ }^{* * *}$ E-mail: reinaldo.lucena@ufms.br.
\end{abstract}

Resumo. A hipótese da aparência ecológica busca entender a dinâmica de uso que uma determinada espécie possui por meio de sua disponibilidade em áreas de vegetação. De acordo com essa hipótese, as plantas aparentes são as mais coletadas e utilizadas pelas pessoas e foi testada na comunidade rural de Cachoeira, município de Brasileira, no Estado do Piauí, Nordeste do Brasil. Calculou-se o valor de uso (VU) para cada espécie. Para o inventário fitossociológico, adotou-se o método pontoquadrante, traçando-se 50 transectos distribuídos nas áreas de vegetação da comunidade, registrando as medidas de perímetro e altura de 2.200 plantas. Realizaram-se entrevistas com os moradores, totalizando 27 informantes (13 homens e 14 mulheres), e registraram-se 34 espécies, 32 gêneros e 20 famílias. As espécies citadas foram agrupadas em categorias de uso. 0 coeficiente de correlação de Spearman foi utilizado para correlacionar os dados fitossociológicos e etnobotânicos. Os valores de uso das espécies e as categorias de uso não se correlacionaram com os parâmetros fitossociológicos. Os resultados da presente pesquisa sugerem que a seleção e utilização de recursos vegetais na região do entorno do Parque Nacional (PARNA) Sete Cidades pode estar relacionada a outros fatores, sendo diferente do encontrado em outros estudos, que relacionam o uso a disponibilidade do recurso. Talvez o fato da comunidade encontrasse no entorno de uma área de preservação
Recebido

03/09/2021

Aceito

$05 / 01 / 2022$

Disponível on line $31 / 01 / 2022$

Publicado

$30 / 04 / 2022$

Acesso aberto

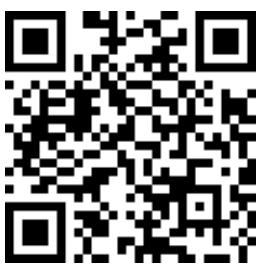

ISSN 2359-1412/RBGAS-2021-0114/2022/9/21/4/59

Rev. Bras. Gest. Amb. Sustent.

http://revista.ecogestaobrasil.net 
ambiental, esteja determinando essa relação, podendo fazer com que essas populações busquem seus recursos em áreas mais distante dessa unidade de conservação.

Palavras-chave: Etnobotânica; Etnobotânica quantitativa; Populações tradicionais; Unidade de conservação.

\begin{abstract}
Can the Ecological Appearance Hypothesis explain the local importance of plant resources in the National Park Sete Cidades region, Piauí, Brazil? The ecological apparency hypothesis seeks to understand the dynamics of use that a particular species has through its availability in vegetation areas. According to this hypothesis, apparent plants are the most collected and used by humans. This hypothesis was tested in the Rural Community of Cachoeira, Municipality of Brasileira, in State of Piauí, Northeast Brazil. We calculated the use value (UV) for each species. For the phytosociological inventory, we adopted the point-quadrant method, plotting 50 transect distributed in the vegetation areas of the community, registering the perimeter measurements and height of 2,200 plants. Interviews wereconducted with householders, totaling 27 informants (13 men and 14 women), and 34 species, 32 genera, and 20 families were recorded. The cited species were grouped into utility categories. The Spearman correlation coefficient was used to correlate phytosociological and ethnobotanical data. The use values of the species and the use categories did not correlate with phytosociological parameters. Regarding the use categories, there were positive correlations frequency). The results of the present research suggest that the selection and use of plant resources in the region surrounding National Park Sete Cidades may be related to other factors, different from what was found in other studies, which relate the use to the availability of the resource. Perhaps the fact that the community found it in the vicinity of an environmental preservation area is determining this relationship, and may make these populations seek their resources in areas further away from this conservation unit.
\end{abstract}

Keywords: Ethnobotany; Quantitative ethnobotany; Traditional populations; Conservation unit.

\footnotetext{
ORCID

D) 0000-0002-1686-1257

Derly Pereira

Brasileiro

(C) 0000-0003-4758-7171

Ezequiel da Costa

Ferreira

(1) 0000-0002-9017-1457

Suellen da Silva Santos

(1) 0000-0003-3847-1249

Thamires Kelly Nunes

Carvalho

(1) 0000-0002-1937-0066

João Everthon da Silva

Ribeiro

(1) 0000-0001-9767-5546

Roseli Farias Melo de

Barros

(1) 0000-0002-5126-8969

Camilla Marques de

Lucena

(1) $0000-0002-1195-4315$

Reinaldo Farias Paiva

de Lucena
}

\section{Introdução}

Os estudos etnobotânicos, nas últimas décadas, vêm buscando entender e investigar as relações existentes entre o conhecimento, uso e disponibilidade de recursos naturais por populações tradicionais, enfocando a conservação da biodiversidade, sob várias perspectivas, em diversas partes do mundo (Albuquerque e Lucena 2005; Sher et al., 2011; Avohou et al., 2012; Singh et al., 2012; Sop et al., 2012; Uprety et al., 2012; Ahmed et al., 2013; Lucena et al., 2013; Murad et al., 2013; Beltrán-Rodríguez et al., 2014; Casas et al., 2016; Rodríguez et al., 2018; Lima-Nascimento et al., 2019; Hassan et al., 2020; Muniz et al., 2021). Esses estudos vêm ocorrendo principalmente em função do aumento 
das ações antrópicas em fragmentos florestais, influência da manutenção do conhecimento tradicional e ocidentalização cultural (Lucena et al., 2014).

Nesse contexto, a partir da década de 1990, houve aumentos das pesquisas etnobotânicas com foco quantitativo, com o emprego de parâmetros estatísticos, índices e teste de hipóteses utilizados para compreender o uso e conhecimento de espécies vegetais nas regiões tropicais (Gaoue et al., 2017), pois essas apresentam uma ampla diversidade de recursos com elevado potencial econômico, indo desde os madeireiros aos não madeireiros (Souza et al., 2018; Andrade et al., 2019). Assim, são necessários estudos que busquem analisar o conhecimento e uso das espécies, bem como averiguar a pressão antrópica que ocorre com a retirada dos recursos vegetais, com implicações para a conservação, e perspectivas para observar a frequência de coleta e distribuição da vegetação local.

Diante disso, surgiram hipóteses ecológicas que auxiliaram no desenvolvimento de estudos etnobotânicos, de modo a buscar entender a dinâmica de uso das plantas por populações tradicionais. Dentre essas hipóteses, destaca-se a hipótese da aparência ecológica, a qual foi proposta, originariamente, para explicar a herbivoria a partir da relação entre os insetos e as plantas (Fenny, 1976; Rhoades e Cates, 1976). Segundo os autores dessa hipótese, as plantas pertencem a dois grupos, sendo as plantas aparentes, que são facilmente encontradas pelos herbívoros em função do elevado porte (árvores, arbustos e grandes herbáceas), e as plantas não aparentes, que dificilmente são encontradas pelos herbívoros, possuindo menor porte (herbáceas e plantas em estágios iniciais de sucessão). Assim, as plantas "aparentes" exigem uma maior procura pelos herbívoros, sendo as mais consumidas, em comparação com as plantas "não aparentes".

Seguindo o pressuposto desta hipótese, Phillips e Gentry (1993a,b) realizaram uma adaptação para que a mesma fosse utilizada em estudos etnobotânicos, substituindo o papel dos herbívoros pelo das populações tradicionais. Desse modo, assumiram que as pessoas tendem a buscar recursos de acordo com a maior visibilidade e disponibilidade na vegetação, ou seja, as plantas que são facilmente visíveis (plantas aparentes) são as mais utilizadas pelas populações tradicionais (Phillips e Gentry, 1993a,b). Esses autores, para testar a hipótese da aparência ecológica, empregaram o índice quantitativo do valor de uso, em que se dá a partir de entrevistas com populações tradicionais sobre a utilização das espécies vegetais, evidenciando a importância dessas plantas para as pessoas. Inicialmente, os estudos testando essa hipótese foram desenvolvidos em florestas úmidas, com resultados positivos, porém Albuquerque et al. (2005) testaram-na pela primeira vez a hipótese em florestas secas (área de Caatinga), buscando entender a relação entre o uso de espécies vegetais locais e sua disponibilidade na vegetação, encontrando resultados diferentes dos realizados em áreas úmidas.

A hipótese da aparência ecológica foi testada por outros pesquisadores em áreas de Caatinga (Albuquerque et al., 2012; Lucena et al., 2012; Lucena et al., 2014; Ribeiro et al., 2014a,b; Lima et al., 2016), e de Cerrado (Lima et al., 2012; Pinho Júnior et al., 2013; Tunholi et al., 2013; Oliveira et al., 2019). Quando testada nessas regiões, tem apresentado resultados distintos em comparação com os das florestas úmidas, em que os resultados foram positivos em relação ao uso e disponibilidade dos recursos vegetais locais. Diante disso, necessita-se de novos estudos em áreas de Caatinga e de Cerrado para observar e tentar compreender melhor a relação entre as populações tradicionais e os recursos vegetais disponíveis, bem como para registrar as espécies que podem sofrer maior pressão antrópica nessas regiões.

Assim, o objetivo deste artigo foi testar a hipótese da aparência ecológica em uma área no entorno do Parque Nacional de Sete Cidades, de forma a buscar respostas sobre o uso e disponibilidade de recursos vegetais nessa região. Para isso, foi assumido que a hipótese de que o uso de espécies lenhosas nativas da região do entorno do PARNA de Sete 
Cidade no Piauí (Nordeste do Brasil) é influenciado pela disponibilidade destas espécies na vegetação local.

\section{Material e métodos}

\section{0 contexto regional e local de trabalho}

Parque Nacional (PARNA) de Sete Cidades foi criado pelo Decreto no 50.744/1961 (Brasil, 1961), localizando-se no nordeste do Estado do Piauí, com sua abrangência territorial sendo distribuída entre os Municípios de Piracurura e Brasileira (Figura 1). De acordo com Cavalcante (2013), a criação desse PARNA "foi reconhecida como nicho prioritário para a conservação da biodiversidade das fitofisionomias de cerrado e caatinga, de sorte que ao longo do tempo as mais diversas pesquisas têm sido ali desenvolvidas". 0 PARNA compreende um mosaico de fitofisionomias, sendo possível observar formações savânicas (Cerrado típico e Cerrado rupestre), formações florestais (Cerradão, Mata Seca Semidecídua e Mata de Galeria Inundável) e formação campestre (Campo Limpo) (Oliveira et al., 2017).

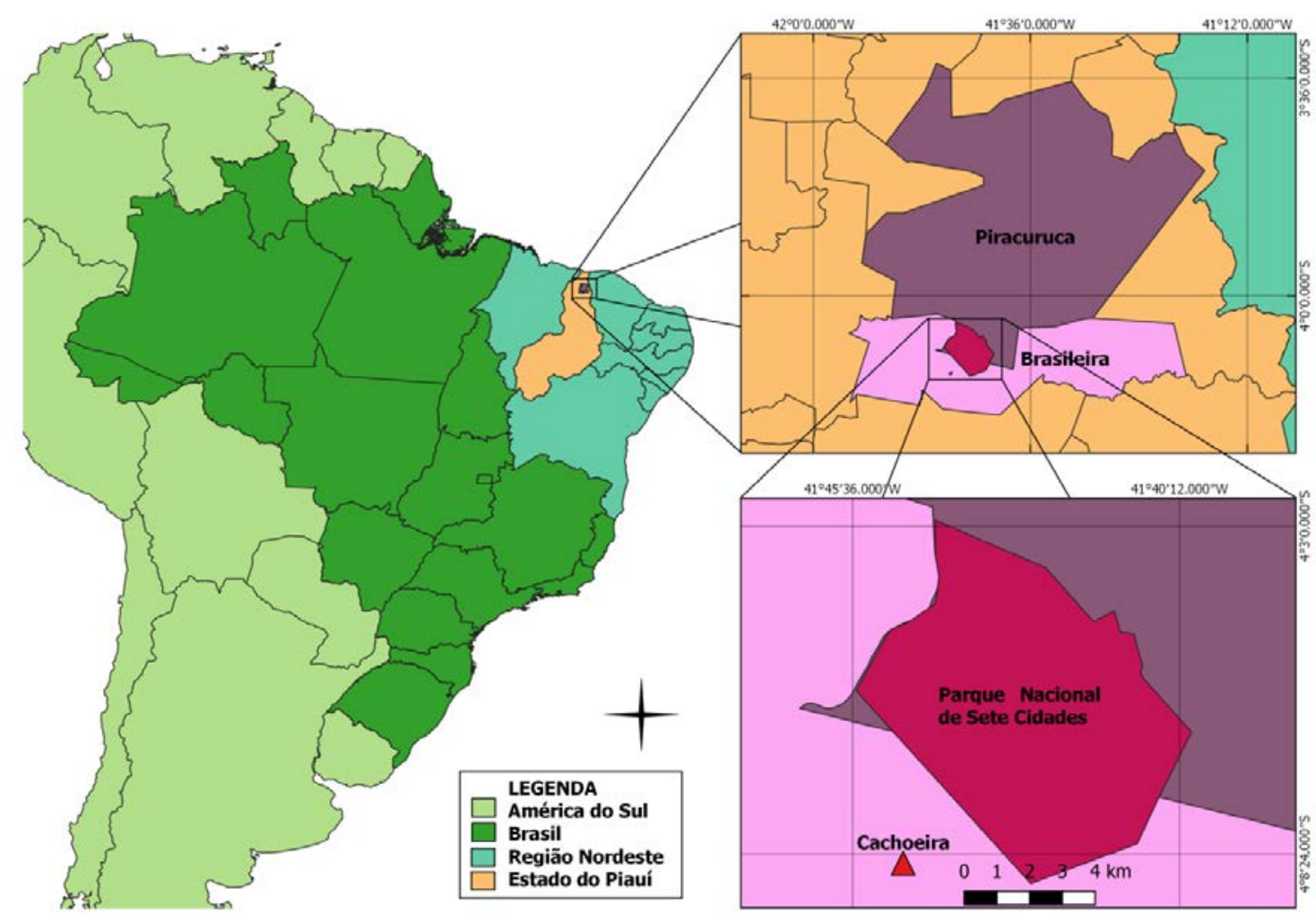

Figura 1. Localização da Comunidade Rural Cachoeira e do Parque Nacional de Sete Cidades, Estado do Piauí, Nordeste, Brasil. Mapa: Ezequiel da Costa Ferreira (2019).

Seu clima é do "tipo subúmido com grande deficiência de água, quarto megatérmico e pequena amplitude térmica anual. Há duas estações bem definidas (seca e chuvosa) durante o ano, com temperaturas médias anuais superiores a $25^{\circ} \mathrm{C}$ e precipitação média anual de 1.558 mm" (Cavalcante, 2013). 
0 estudo foi realizado na Comunidade Rural Cachoeira, pertencente ao Município de Brasileira ( $4^{\circ} 7^{\prime} 51^{\prime \prime} \mathrm{S}, 41^{\circ} 46^{\prime} 55^{\prime \prime} \mathrm{W}$ ) (Geohack, 2019), tendo cerca de 20 famílias, distando aproximadamente $10 \mathrm{~km}$ da sede do PARNA. A escolha desta comunidade deu-se pelo fato de estar nas proximidades do PARNA, na borda da zona de amortecimento, e pela constatação do uso de recursos da vegetação local pelas pessoas. A região escolhida apresenta um mosaico de fitofisionomias, sendo possível observar formações savânicas (Cerrado típico e Cerrado rupestre), formações florestais (Cerradão, Mata Seca Semidecídua e Mata de Galeria Inundável) e formação campestre (Campo Limpo) (Oliveira et al., 2017). A precipitação média anual é de $1.557,8 \mathrm{~mm}$ e a temperatura média anual é de $26,5^{\circ} \mathrm{C}$ (Oliveira et al., 2017).

\section{Amostragem da vegetação}

Para testar a hipótese da aparência ecológica, realizou-se um inventário fitossociológico em áreas de vegetação no entorno do Parque Nacional de Sete Cidades, nas proximidades da Comunidade Cachoeira, por meio do qual registraram-se as espécies consideradas úteis pela população local.

No inventário fitossociológico foi adotado o Método do Ponto-Quadrante (Cottam e Curtis, 1956), sendo distribuídos 50 transectos, cada um com $100 \mathrm{~m}$ de comprimento. 0 local foi indicado pelos mateiros locais como área de possível coleta de plantas pelos moradores locais, estando a área de amostragem fitossociológica plotada num raio de $4 \mathrm{~km}$ da comunidade. Todos os transectos foram dispostos paralelamente uns aos outros, com distância entre eles de $10 \mathrm{~m}$. Ao longo de cada transecto, 11 pontos foram marcados (ponto zero até o ponto 10), com distância de $10 \mathrm{~m}$ entre cada um. Em cada ponto, uma cruz de madeira foi utilizada para indicar o direcionamento de quatro espécimes lenhosas a serem aferidas, formando um quadrante em cada ponto do transecto. Todos os indivíduos tiveram suas alturas e diâmetros dos caules no nível do solo (DNS) $\geq 3 \mathrm{~cm}$ verificados e anotados em uma planilha. Por meio desse delineamento amostral, foram contabilizados 2.200 indivíduos distribuídos em 5 ha de área de vegetação. Foram coletadas amostras das plantas inventariadas para identificação, tombamento, herborização e tombamento na forma de exsicatas no Herbário Jaime Côelho de Moraes (EAN), no Centro de Ciências Agrárias (CCA), da Universidade Federal da Paraíba (UFPB). A organização das famílias seguiu o APG IV (2016), brm como o nome das espécies e a abreviatura do nome dos autores seguem conforme o estabelecido na Lista de Espécies da Flora do Brasil (2020).

Foram adotados os seguintes parâmentos fitossociologicos: densidade relativa, dominância relativa e frequência relativa, sendo analisados de acordo com Araújo e Ferraz (2010). A Densidade Relativa (DRt, \%), foi estimada pelo número de indivíduos de um determinado táxon com relação ao total de indivíduos amostrados. Frequência Relativa (FRt, \%) foi estimada com base na FAt (Frequência absoluta da espécie em questão), em relação à Frequência Total (FT, \%), que representa o somatório de todas as frequências absolutas. A Dominância Relativa (DoRt,\%) representa a porcentagem de DoA (dominância absoluta da espécie em questão) com relação à dominância total (DoT).

\section{Inventário etnobotânico}

0 presente estudo foi desenvolvido em dois momentos distintos, entre maio e junho, e entre novembro e dezembro de 2018, no qual participaram 27 mantenedores familiares, sendo 13 homens e 14 mulheres, da comunidade rural Cachoeira, a qual localiza-se no entorno do Parque Nacional de Sete Cidades. Essas pessoas foram informadas sobre o objetivo do estudo para que pudessem assinar o Termo de Consentimento Livre e Esclarecido (TCLE), exigido pelo Conselho Nacional de Saúde (Resolução CNS no 466/2012), por meio do Comitê de Ética em Pesquisa com Seres 
Humanos (CEP), do Hospital Lauro Wanderley, da Universidade Federal da Paraíba (CEP/HULW/UFPB), com Parecer Consubstanciado no 3.064.256.

0 formulário semiestruturado utilizado nas entrevistas apresentou perguntas específicas sobre as espécies vegetais conhecidas e utilizadas pelos moradores, buscando reconhecer e registrar todas as utilidades de cada espécie, sendo as citações de uso organizadas em categorias de acordo com a literatura especializada (Lucena et al., 2012a), sendo elas: alimentação, combustível, construção, forragem, medicinal, tecnologia, veneno/abortiva, veterinária, mágico-religioso, ornamentação, dentre outras. Na categoria outros usos foram incluídas as citações para higiene pessoal (lavar cabelos, escovar dente, etc.), bioindicadores (sinal de chuva) e sombra, ou outra.

\section{Análise dos dados fitossociológicos e da hipótese da aparência ecológica}

Foi utilizado o coeficiente de Correlação de Spearman para testar a relação entre o valor de uso (VU) e para a disponibilidade das plantas utilizou-se o programa BioEstat 5.0 (Sokal e Rholf, 1995). Foi observado se existia relação entre o VU com os parâmetros fitossociológicos (dominância relativa, frequência relativa e densidade relativa). Para a análise por categorias de uso, foram incluídas as espécies que obtiveram alguma citação de uso para a categoria em questão.

\section{Análises dos dados etnobotânicos}

No teste da hipótese da aparência ecológica foram consideradas na análise dos dados etnobotânicos somente as espécies mencionadas nas entrevistas como úteis, e que foram registradas no levantamento fitossociológico.

Para cada espécie e categorias de uso calculou-se, respectivamente, o seu valor de uso pelas fórmulas $\mathrm{VU}=\sum \mathrm{Ui} / \mathrm{n}$, e VUc $=\sum \mathrm{VU} / \mathrm{nc}$, descritas por Rossato et al. (1999) onde: $\mathrm{Ui}=$ número de usos mencionados por cada informante, $\mathrm{n}=$ número total de informantes, VUc $=$ valor de uso de cada espécie na categoria, nc = número de espécies na categoria.

\section{Resultados}

\section{Amostragem da vegetação}

Registraram-se 34 espécies úteis, pertencentes a 32 gêneros e 20 famílias botânicas. Campomanesia velutina (Cambess.) O. Berg. (guabiraba-preta) predominou com 288 indivíduos, seguido por Copaifera luetzelburgii Harms (podói) (262 indivíduos) e Aspidosperma sp (pequiá) (187 indivíduos). As famílias com maior ocorrência foram Fabaceae (339 indivíduos), Myrtaceae (330 indivíduos) e Rubiaceae (203 indivíduos) (Tabela 1).

As espécies em destaque quanto a Densidade Relativa foram Campomanesia velutina (guabiraba-preta) (DeR $=13,09)$, Copaifera luetzelburgii (podói) $(\mathrm{DeR}=11,91) \mathrm{e}$ Cordiera sessilis (Vell.) Kuntze (maria preta) (DeR =9,23). Quanto à dominância relativa, destacaram-se Copaifera luetzelburgii (podói) (DoR = 17,21), Aspidosperma sp. (pequiá) $($ DoR $=8,96)$ e Campomanesia velutina (guabiraba-preta) $($ DoR $=8,67)$. Já para a frequência relativa (FRt) destacaram-se Copaifera luetzelburgii (podói) (FRt $=11,83$ ), Campomanesia velutina (guabiraba preta) (FRt $=11,72$ ) e Cordiera sessilis (maria-preta) $(\mathrm{FRt}=8,72)$.

\section{Inventário etnobotânico}

Registraram-se 34 espécies úteis nas entrevistas, pertencentes a 32 gêneros e 20 famílias botânicas (Tabela 1). Observa-se uma grande variação entre os maiores e menores valores de uso para as espécies. As espécies que apresentaram maior VU foram Aspidosperma sp. (pequiá) (VU = 5), Hymenaea courbaril L. (jatobá) (VU = 3,11), Ximenia americana L. (ameixa) (VU = 2,67), Myracrodruon urundeuva Allemão (aroeira) (VU = 
2,18) e Terminalia argentea Mart. (Cascudo) (VU = 2,11), Campomanesia velutina (guabiraba-preta) (VU =1,33). Das 34 espécies, apenas seis obtiveram valor de uso maior do que 1,00, o que pode indicar uma concentração maior de usos sobre as mesmas plantas.

Tabela 1. Espécies e famílias registradas no levantamento etnobotânico, seguidas de suas respectivas categorias de uso e partes utilizadas e dados fitossociológicos evidenciados na comunidade Cachoeira, município de Brasileira, Piauí, Nordeste do Brasil. Al = Alimento; $\mathrm{Cb}=$ Combustível; $\mathrm{Ct}=$ Construção; $\mathrm{Fr}=$ Forragem; $\mathrm{Hi}=$ Higiene pessoal; $\mathrm{Mr}=$ Mágico-religioso; $\mathrm{Me}=$ Medicinal; Or = Ornamental; So = Sombra; Tc = Tecnologia; Va = Veneno-Abortivo; Vt = Veterinário. $\mathrm{Ca}=$ Casca $; \mathrm{Ec}=$ Entrecasca; $\mathrm{Fo}=$ Folha; Fl = Flor; Fr = Fruto; La = Látex; $\mathrm{Ma}=$ Madeira; $\mathrm{Pc}=$ Planta Completa; $\mathrm{Ra}=$ Raiz; Se = Semente; Re = Resina. VU = Valor de uso; $\mathrm{N}^{\circ}$ Ind = Número de indivíduos; VI = valor de importância; DeR = Densidade relativa; DoR = Dominância relativa; FRt = Frequência relativa.

\begin{tabular}{|c|c|c|c|c|c|c|c|}
\hline $\begin{array}{l}\text { Família / Espécie/ Nome } \\
\text { vernacular }\end{array}$ & Usos & $\begin{array}{c}\text { Partes } \\
\text { utilizadas }\end{array}$ & VU & $\mathbf{N}^{\circ}$ Ind & DeR & DoR & FRt \\
\hline \multicolumn{8}{|l|}{ Anacardiaceae } \\
\hline $\begin{array}{l}\text { Anacardium humile A. St.-Hil. } \\
\text { (cajuí) }\end{array}$ & $\begin{array}{c}\mathrm{Al}, \mathrm{Cb}, \mathrm{Fr} \\
\mathrm{Me}, \mathrm{Vt}\end{array}$ & $\begin{array}{l}\text { Ca, Ec, Fr, } \\
\text { Ma }\end{array}$ & 0,85 & 6 & 0,27 & 1,21 & 0,33 \\
\hline $\begin{array}{l}\text { Astronium fraxinifolium Schott. } \\
\text { (aroeira-gonçalave) }\end{array}$ & $\begin{array}{l}\mathrm{Cb}, \mathrm{Ct}, \mathrm{Me}, \\
\mathrm{Vt}\end{array}$ & Ec, Ma & 0,89 & 2 & 0,09 & 0,48 & 0,11 \\
\hline $\begin{array}{l}\text { Myracrodruon urundeuva } \\
\text { Allemão (aroeira) }\end{array}$ & $\begin{array}{l}\mathrm{Cb}, \mathrm{Ct}, \mathrm{Me}, \\
\mathrm{Va}, \mathrm{Ve}\end{array}$ & $\mathrm{Ca}, \mathrm{Ec}, \mathrm{Ma}$ & 2,18 & 36 & 1,64 & 1,71 & 1,94 \\
\hline $\begin{array}{l}\text { Tapirira guianensis Aubl. } \\
\text { (pau-pombo) }\end{array}$ & $\mathrm{Ct}, \mathrm{Tc}$ & $\mathrm{Ma}$ & 0,22 & 6 & 0,27 & 0,29 & 0,22 \\
\hline \multicolumn{8}{|l|}{ Annonaceae } \\
\hline $\begin{array}{l}\text { Duguetia echinophora R.E.Fr. } \\
\text { (ata-brava) }\end{array}$ & $\mathrm{Ct}$ & $\mathrm{Ma}$ & 0,04 & 2 & 0,09 & 0,51 & 0,11 \\
\hline Ephedranthus sp. (cunduru) & $\begin{array}{l}\mathrm{Al}, \mathrm{Cb}, \mathrm{Ct}, \\
\mathrm{Fr}\end{array}$ & Fr, Ma & 0,52 & 63 & 2,86 & 4,59 & 3,05 \\
\hline \multicolumn{8}{|l|}{ Apocynaceae } \\
\hline $\begin{array}{l}\text { Aspidosperma multiflorum A. } \\
\text { DC. (pequiá-branco) }\end{array}$ & $\mathrm{Cb}, \mathrm{Ct}, \mathrm{Tc}$ & $\mathrm{Ma}$ & 0,18 & 1 & 0 & 0,02 & 0,06 \\
\hline Aspidosperma sp. (pequiá) & $\begin{array}{l}\mathrm{Cb}, \mathrm{Ct}, \mathrm{Fr} \\
\mathrm{Tc}\end{array}$ & Fo, Ma & 5 & 187 & 8,5 & 8,96 & 8,33 \\
\hline \multicolumn{8}{|l|}{ Arecaceae } \\
\hline $\begin{array}{l}\text { Bactris campestres Poepp. ex } \\
\text { Mart. (tucum) }\end{array}$ & $\begin{array}{c}\mathrm{Al}, \mathrm{Ct}, \mathrm{Fr}, \\
\mathrm{Tc}\end{array}$ & $\begin{array}{c}\text { Fo, Fr, Ma, } \\
\text { Se }\end{array}$ & 0,85 & 2 & 0,002 & 0,16 & 0,11 \\
\hline Capparaceae & & & & & & & - \\
\hline $\begin{array}{l}\text { Cynophalla flexuosa (L.) Presl. } \\
\text { (jacaré) }\end{array}$ & Fr & Fr & 0,04 & 120 & 5,45 & 2,88 & 5,44 \\
\hline \multicolumn{8}{|l|}{ Combretaceae } \\
\hline $\begin{array}{l}\text { Combretum laxum Jack. } \\
\text { (rama-branca) }\end{array}$ & $\mathrm{Cb}, \mathrm{Ct}$ & $\mathrm{Ma}$ & 0,15 & 17 & 0,77 & 0,79 & 0,89 \\
\hline $\begin{array}{l}\text { Terminalia argentea Mart. } \\
\text { (cascudo) }\end{array}$ & $\begin{array}{l}\mathrm{Cb}, \mathrm{Ct}, \mathrm{Me}, \\
\mathrm{Tc}\end{array}$ & $\mathrm{Ca}, \mathrm{Ec}, \mathrm{Ma}$ & 2,11 & 15 & 0,68 & 2,09 & 0,83 \\
\hline \multicolumn{8}{|l|}{ Dilleniaceae } \\
\hline $\begin{array}{l}\text { Curatella americana } \mathrm{L} . \\
\text { (sambaíba) }\end{array}$ & $\mathrm{Ct}, \mathrm{Tc}$ & $\mathrm{Ma}$ & 0,11 & 2 & 0,09 & 0,12 & 0,11 \\
\hline \multicolumn{8}{|l|}{ Euphorbiaceae } \\
\hline $\begin{array}{l}\text { Croton adamantinus Mull. Arj. } \\
\text { (marmeleiro) }\end{array}$ & $\mathrm{Me}, \mathrm{Vt}$ & $\mathrm{Ca}, \mathrm{Fo}$ & 0,22 & 11 & 0,5 & 0,1 & 0,56 \\
\hline
\end{tabular}


Tabela 1. Continuação.

\begin{tabular}{|c|c|c|c|c|c|c|c|}
\hline $\begin{array}{l}\text { Família / Espécie/ Nome } \\
\text { vernacular }\end{array}$ & Usos & $\begin{array}{c}\text { Partes } \\
\text { utilizadas } \\
\end{array}$ & VU & $\mathbf{N}^{\circ}$ Ind & DeR & DoR & FRt \\
\hline \multicolumn{8}{|l|}{ Fabaceae } \\
\hline $\begin{array}{l}\text { Anadenanthera colubrina (Vell.) } \\
\text { Brenan (angico-preto) }\end{array}$ & $\mathrm{Ct}, \mathrm{Me}, \mathrm{Vt}$ & $\mathrm{Ca}, \mathrm{Ec}, \mathrm{Ma}$ & 0,67 & 1 & 0,05 & 0,02 & 0,06 \\
\hline $\begin{array}{l}\text { Bauhinia dubia G. Dori } \\
\text { (mororó) }\end{array}$ & $\mathrm{Cb}, \mathrm{Ct}, \mathrm{Fr}$ & Fo, Ma & 0,52 & 13 & 1,27 & 0,22 & 1,44 \\
\hline $\begin{array}{l}\text { Copaifera luetzelburgii Harms } \\
\text { (podói) }\end{array}$ & $\begin{array}{l}\mathrm{Al}, \mathrm{Cb}, \mathrm{Ct}, \\
\mathrm{Fr}\end{array}$ & Fr, Ma & 0,85 & 262 & 11,91 & 17,21 & 11,83 \\
\hline Hymenaea courbaril L. (jatobá) & $\begin{array}{c}\mathrm{Al}, \mathrm{Cb}, \mathrm{Ct}, \\
\mathrm{Fr}, \mathrm{Mr}, \mathrm{Me}, \\
\text { Or, Tc }\end{array}$ & $\begin{array}{l}\text { Ca, Ec, Fr, } \\
\text { Ma, Re }\end{array}$ & 3,11 & 10 & 0,45 & 0,63 & 0,5 \\
\hline $\begin{array}{l}\text { Parkia platycephala Benth. } \\
\text { (faveira) }\end{array}$ & $\begin{array}{c}\mathrm{Cb}, \mathrm{Fr}, \mathrm{So} \\
\mathrm{Va}\end{array}$ & $\mathrm{Fr}, \mathrm{Ma}$ & 0,59 & 18 & 0,82 & 3,09 & 0,94 \\
\hline $\begin{array}{l}\text { Plathymenia reticulata Benth. } \\
\text { (candeia) }\end{array}$ & $\begin{array}{l}\mathrm{Cb}, \mathrm{Ct}, \mathrm{Me}, \\
\mathrm{Tc} \\
\end{array}$ & $\mathrm{Ca}, \mathrm{Ma}$ & 0,92 & 2 & 0,09 & 0,09 & 0,11 \\
\hline $\begin{array}{l}\text { Vatairea macrocarpa (Benth.) } \\
\text { Ducke (amargoso) }\end{array}$ & $\begin{array}{l}\mathrm{Cb}, \mathrm{Ct}, \mathrm{Me}, \\
\mathrm{Or}, \mathrm{Tc} \\
\end{array}$ & Fr, Ma, Se & 0,59 & 33 & 1,5 & 2,72 & 1,67 \\
\hline \multicolumn{8}{|l|}{ Lamiaceae } \\
\hline $\begin{array}{l}\text { Vitex polygama Cham. } \\
\text { (mama-cachorra) }\end{array}$ & $\mathrm{Al}, \mathrm{Cb}, \mathrm{Fr}$ & $\mathrm{Fr}, \mathrm{Ma}$ & 0,18 & 1 & 0,05 & 0,09 & 0,06 \\
\hline \multicolumn{8}{|l|}{ Malpighiaceae } \\
\hline Byrsonima sp (murici) & $\begin{array}{l}\mathrm{Al}, \mathrm{Cb}, \mathrm{Ct}, \\
\mathrm{Fr}\end{array}$ & Fr, Ma & 0,37 & 32 & 1,45 & 1,83 & 1,5 \\
\hline \multicolumn{8}{|l|}{ Malvaceae } \\
\hline $\begin{array}{l}\text { Luehea speciosa Willd. } \\
\text { (açoita-cavalo) }\end{array}$ & $\mathrm{Me}, \mathrm{Vt}$ & $\mathrm{Ca}, \mathrm{Ec}$ & 0,22 & 24 & 1,09 & 0,65 & 1,11 \\
\hline \multicolumn{8}{|l|}{ Melastomataceae } \\
\hline Mouriri samanensis Urb. (criuli) & $\mathrm{Fr}$ & Fr & 0,04 & 1 & 0,05 & 0,01 & 0,06 \\
\hline \multicolumn{8}{|l|}{ Myrtaceae } \\
\hline $\begin{array}{l}\text { Campomanesia aromatica } \\
\text { (Aubl.) Griseb } \\
\text { (guabiraba-amarela) }\end{array}$ & $\begin{array}{l}\mathrm{Al}, \mathrm{Cb}, \mathrm{Ct} \\
\mathrm{Fr}\end{array}$ & $\mathrm{Fr}, \mathrm{Ma}$ & 0,48 & 38 & 1,73 & 2,49 & 1,83 \\
\hline $\begin{array}{l}\text { Campomanesia velutina } \\
\text { (Cambess.) 0. Berg. } \\
\text { (guabiraba-preta) }\end{array}$ & $\begin{array}{l}\mathrm{Al}, \mathrm{Cb}, \mathrm{Ct} \\
\mathrm{Fr}, \mathrm{Me}(5)\end{array}$ & $\begin{array}{l}\text { Ec, Fr, Ma, } \\
\quad \mathrm{Se}\end{array}$ & 1,33 & 288 & 13,09 & 8,67 & 11,72 \\
\hline Myrcia rostrata DC. (canelinha) & $\mathrm{Cb}, \mathrm{Fr}$ & Fr, Ma & 0,07 & 4 & 0,18 & 0,06 & 0,22 \\
\hline \multicolumn{8}{|l|}{ Opiliaceae } \\
\hline $\begin{array}{l}\text { Agonandra brasiliensis Miers ex } \\
\text { Benth. \& Hook. f. (marfim) }\end{array}$ & $\begin{array}{l}\mathrm{Al}, \mathrm{Cb}, \mathrm{Ct} \\
\mathrm{Fr}, \mathrm{Tc}(5) \\
\end{array}$ & Fr, Ma, Se & 0,4 & 3 & 0,14 & 0,05 & 0,17 \\
\hline \multicolumn{8}{|l|}{ Rubiaceae } \\
\hline $\begin{array}{l}\text { Cordiera sessilis (Vell.) Kuntze } \\
\text { (maria-preta) }\end{array}$ & $\begin{array}{l}\mathrm{Al}, \mathrm{Cb}, \mathrm{Ct}, \\
\mathrm{Fr}\end{array}$ & $\mathrm{Fr}, \mathrm{Ma}$ & 0,18 & 203 & 9,23 & 4,88 & 8,72 \\
\hline \multicolumn{8}{|l|}{ Sapindaceae } \\
\hline $\begin{array}{l}\text { Magonia pubescens A. St.-Hil. } \\
\text { (tingui) }\end{array}$ & $\mathrm{Cb}, \mathrm{Ct}, \mathrm{Tc}$ & $\mathrm{Ma}, \mathrm{Fr}$ & 0,18 & 7 & 0,32 & 0,75 & 0,39 \\
\hline \multicolumn{8}{|l|}{ Sapotaceae } \\
\hline $\begin{array}{l}\text { Pouteria ramiflora (Mart.) } \\
\text { Radlk. (pitomba-de-leite) }\end{array}$ & $\begin{array}{l}\mathrm{Al}, \mathrm{Ct}, \mathrm{Fr} \text {, } \\
\text { So }\end{array}$ & $\mathrm{Ma}, \mathrm{Fr}$ & 0,29 & 33 & 1,5 & 1,26 & 1,78 \\
\hline \multicolumn{8}{|l|}{ Vochysiaceae } \\
\hline $\begin{array}{l}\text { Salvertia convallariodora A. } \\
\text { St.-Hil. (folha-larga) }\end{array}$ & Or & Pc & 0,04 & 2 & 0,09 & 0,8 & 0,11 \\
\hline
\end{tabular}


Tabela 1. Continuação.

\begin{tabular}{|l|c|c|c|c|c|c|c|}
\hline $\begin{array}{l}\text { Família / Espécie/ Nome } \\
\text { vernacular }\end{array}$ & Usos & $\begin{array}{c}\text { Partes } \\
\text { utilizadas }\end{array}$ & VU & $\mathbf{N}^{\circ}$ Ind & DeR & DoR & FRt \\
\hline Ximeniaceae & & & & & & & \\
\hline Ximenia americana L. (ameixa) & $\begin{array}{c}\mathrm{Al}, \mathrm{Cb}, \mathrm{Fr}, \\
\mathrm{Me}, \mathrm{Va}, \mathrm{Vt}\end{array}$ & $\begin{array}{c}\mathrm{Ca}, \mathrm{Ec}, \mathrm{Fr}, \\
\mathrm{Ma}, \mathrm{Re}\end{array}$ & 2,67 & 4 & 0,18 & 0,11 & 0,22 \\
\hline
\end{tabular}

\section{Hipótese da Aparência Ecológica}

Não houve correlação entre os parâmetros fitossociológicos e o valor de uso. Dessa forma, a aparência ecológica não explica a relação das pessoas da comunidade estudada com os recursos vegetais disponíveis localmente. Em todos os testes estatísticos realizados entre densidade $x \mathrm{VU}$, frequência $x \mathrm{VU}$, dominância $\mathrm{x} V \mathrm{VU}$, obteve-se $\mathrm{p}>0,005$ (densidade com rs $=0,15 ; \mathrm{p}>0,05$; frequência com rs $=0,15 ; \mathrm{p}>0,05$; dominância com $\mathrm{RS}=0,25 ; \mathrm{p}>0,05)$.

Analisando o valor de uso com cada uma das categorias utilitárias, por meio de teste estatístico, também se observou ausência de correlação, refutando assim a hipótese da aparência ecológica ( $\mathrm{p}>0,05$ em todas as categorias de uso).

\section{Discussão}

As pesquisas que foram realizadas em áreas de florestas úmidas, e testaram a hipótese da aparência ecológica, apresentaram resultados significativos comprovando de forma positiva a relação do uso e disponibilidade de recursos naturais (Phillips e Gentry, 1993a,b; Mutchnick e McCarth, 1997; Galeano, 2000; Ferraz et al., 2002; La Torre-Cuadros e Islebe, 2003; Lawrence et al., 2005; Cunha e Albuquerque, 2006; Thomas et al., 2009; Jiménez-Escobar e Rangel-Ch, 2012), sendo mais robustos dos que os estudos conduzidos nas florestas secas (Albuquerque et al., 2005; Ferraz et al., 2006; Lucena et al., 2007; Balcázar, 2012; Lucena et al., 2012a,b; Lucena et al., 2014; Ribeiro et al., 2014a,b; Trindade et al., 2015; Lima et al., 2016).

Comparando os dados etnobotânicos (valor de uso) com os ecológicos (parâmetros fitossociológicos), observou-se na Comunidade Cachoeira, localizada na região do entorno do Parque Nacional de Sete Cidades no Piauí, que não houve correlação do valor de uso com a dominância relativa, frequência relativa e densidade relativa. Nesse caso, refutando a hipótese da aparência ecológica na comunidade estudada. Diferente desse resultado, algumas pesquisas realizadas na Caatinga, registraram relações positivas confirmando essa hipótese em seu aspecto ecológico (Lucena et al., 2007; Lucena et al., 2012a,b; Ribeiro et al., 2014a,b; Trindade et al., 2015; Lima et al., 2016), contudo, nessas investigações, que ainda são poucas, se encontraram resultados diferentes e sem um padrão, o que reforça a importância e necessidade de realização de mais pesquisas para o teste e aplicação da hipótese aparência ecológica, buscando assim uma compreensão sobre a dinâmica de uso das plantas úteis no semiárido brasileiro, e em outras florestas secas e outros biomas e ecossistemas brasileiros.

Baseado na realidade ecológica, cultural e socioeconômica das comunidades rurais do Nordeste do Brasil, principalmente na região da Caatinga e do Cerrado, a hipótese da aparência ecológica pode apresentar resultados diferentes. A relação uso e disponibilidade, testada de forma geral nas florestas secas, tem-se revelado com resultados distintos. Os estudos realizados por Albuquerque et al. (2005), em Alagoinha (PE), Ferraz et al. 2006, em Floresta (PE), e por Lucena et al. (2014), em São Mamede (PB), todos localizados em áreas de Caatinga, obtiveram resultados contrários ao esperado pela aparência, os quais observaram que as espécies mais valorizadas pela população são

Rev. Bras. Gest. Amb. Sustent., 2021, vol. 9, n. 21, p. 59-73. 
aquelas que possuem uma disponibilidade local baixa. Já outros estudos (Lucena et al., 2007; Lucena et al., 2012a; Lucena et al., 2012b; Ribeiro et al., 2014b; Guerra et al., 2015; Trindade et al., 2015; Lima et al., 2016), registraram correlações positivas que corroboram com os pressupostos da aparência ecológica. Já no caso da presente pesquisa, a aparência ecológica não apresentou resultado positivo como esses outros estudos. Em contrapartida, a aparência ecológica tem sido ratificada em estudos realizados em florestas úmidas em diversas regiões, principalmente na Amazônia e Mata Atlântica (Paz y Minõ et al., 1991; Mutchnick e McCarthy, 1997; Galeano, 2000; Torre-Cuadros e Islebe, 2003; Lawrence et al., 2005; Cunha et al., 2006).

Pesquisas realizadas em outros países também vêm confirmando a hipótese da aparência ecológica, a exemplo do estudo de Ayantude et al. (2009) na África, no qual comprovaram que as plantas "aparentes" (lenhosas) obtiveram maior importância relativa do que as "não aparentes" (herbáceas), confirmando a relação entre o uso e a disponibilidade dos recursos vegetais, sendo as plantas lenhosas os maiores alvos de coleta pelas populações humanas, principalmente em virtude da facilidade de serem encontradas. Outros autores também argumentam que as áreas mais acessíveis são mais vulneráveis à exploração pelas populações tradicionais (Stagegaard et al., 2002; Lucena et al., 2007), provavelmente essa facilidade de acesso possa ter uma influência direta no teste da hipótese da aparência ecológica.

No presente estudo, já que a aparência ecológica não explicou a relação entre uso e disponibilidade dos recursos vegetais, o que vem determinando a seleção e utilização de espécies úteis nessa região? Por que a aparência ecológica se aplicou de forma positiva em outros estudos realizados em florestas secas, e não no presente estudo?

Como nesse estudo não houve correlações de usos, pode-se supor que a pressão de uso está sendo baixa. Para tal comprovação seria interessante a realização de mais estudos específicos nesta área que visem a entender a relação pessoas/recursos, e como está ocorrendo à relação de uso e disponibilidade do recurso com a dinâmica cultural e socioeconômica da região do entorno do Parque Nacional de Sete Cidades. Talvez o fato da comunidade encontrar-se no entorno de uma área de preservação ambiental, esteja determinando essa relação, podendo fazer com que essas populações busquem seus recursos em áreas mais distante dessa unidade de conservação.

Conforme Lucena et al. (2012), a hipótese da aparência ecológica pode nos fornecer possíveis padrões de usos locais de utilização dos recursos vegetais em áreas de Caatinga, e em outras regiões também, mas como mencionado pelos autores, esta hipótese sofre influência de acordo com os usos atribuídas às espécies por cada informante, além destes, provavelmente os costumes e as dinâmicas de cada comunidade podem também influir nos agrupamentos das categorias para cada espécie, e com usos atribuídos de forma bem específica em cada região.

\section{Conclusão}

A hipótese da aparência ecológica parece distinguir padrões de uso local dos recursos vegetais da Caatinga e do Cerrado. No entanto, o padrão de uso de recursos vegetais pode mudar, dependendo da utilidade que os moradores atribuem a cada espécie em cada região.

Os resultados da presente pesquisa sugerem que a seleção e utilização de recursos vegetais na região do entorno do PARNA de Sete Cidades pode estar relacionada a outros fatores, sendo diferente do encontrado em outros estudos, que relacionam o uso a disponibilidade do recurso. Esses achados apontam para a necessidade de estudos mais aprofundados no sentido de confirmar ou refutar a hipótese da aparência ecológica em regiões de Cerrado e Caatinga, e testar ou propor outras hipóteses que possam explicar os critérios de seleção de plantas lenhosas no nordeste brasileiro. 
0 fato da hipótese da aparência ecológica ter dado nulo, pode também está associado a um efeito prático e à eficiência dos serviços de fiscalização pelos órgãos ambientais locais e regionais. As informações desse estudo mostram a necessidade de avançar com pesquisas em áreas de unidades de conservação, os quais podem contribuir consideravelmente com o Instituto Chico Mendes de Conservação da Biodiversidade ICMBio.

\section{Conflito de interesses}

Os autores declaram não haver conflito de interesses.

\section{Referências}

Ahmed, E.; Arshad, M.; Saboor, A.; Qureshi, R.; Mustafa, G.; Sadiq, S.; Chaudhari, S. K. Ethnobotanical appraisal and medicinal use of plants in Patriata, New Murree, evidence from Pakistan. Journal of Ethnobiology and Ethnomedicine, v. 9, n. 13, 2013. https://doi.org/10.1186/1746-4269-9-13

Albuquerque, U. P.; Andrade, L. H. C.; Silva, A. C. O. Use of plant resources in a seasonal dry forest (Northeastern Brazil). Acta Botanica Brasílica, v. 19, n. 1, p. 27-38, 2005. https://doi.org/10.1590/S0102-33062005000100004

Albuquerque, U. P.; Lucena, R. F. P. Can apparency affect the use of plants by local people in tropical forests? Interciencia, v. 30, n. 8, p. 506-511, 2005.

Albuquerque, U. P.; Araújo, E. L.; El-Deir A. C. A; Lima, A. L. A; Souto A.; Bezerra, B. M.; Ferraz, E. M. N.; Freire, E. M. X.; Sampaio, E. V. S. B.; Las-Casas, F. M. G.; Moura, G. J. B.; Pereira, G. A.; Melo, J. G.; Ramos, M. A.; Rodal, M. J. N.; Schiel, N.; Lyra-Neves, R. M.; Alves, R. R. N.; Azevedo-Júnior, S. M.; Telino Júnior, W. R.; Severi, W. Caatinga revisited: Ecology and conservation of an important seasonal dry forest. The Scientific World Journal, v. 2012, 2012. https://doi.org/10.1100/2012/205182

Andrade, A. M. F.; Alves, C. A. B.; Souza, R. S. S.; Silva, S. Inventário etnobotânico e uso das espécies madeireiras e não madeireiras na Comunidade de Ouricuri, Pilões-PB, Nordeste do Brasil. Revista Equador, v. 8, n. 2, p. 399-421, 2019.

APG IV. An update of the Angiosperm Phylogeny Group classification for the orders Linnean Society, p. 1-20, 2016.

Avohou H. T.; Vodouhe S. R.; Dansi A.; Bellon M.; Kpeki B. Ethnobotanical factors influencing the use and management of wild edible plants in agricultural environments in Benin. Ethnobotany Research \& Applications, v. 10, p. 571-592, 2012.

Ayantude, A. A.; Hiernaux, P.; Briejer, M.; Udo, H.; Tabo, R. Uses of local plant species by agropastoralists in South-Western Niger. Etnobotany Research \& Applications, v. 7, p. 53-66, 2009.

Balcázar, A. L. Hipótese da aparência na dinâmica do uso de plantas medicinais na Floresta Nacional do Araripe (Ceará, Nordeste do Brasil). Recife: UFRPE, 2012. (Dissertação de metrado).

Beltrán-Rodríguez, L.; Ortiz-Sánchez, A.; Mariano, N. A.; Maldonado-Almanza, B.; Reyes-García, V. Factors affecting ethnobotanical knowledge in a mestizo community of the Sierra de Huautla Biosphere Reserve, Mexico. Journal of Ethnobiology and Ethnomedicine, v. 10, n. 14, 2014. https://doi.org/10.1186/1746-4269-10-14 
Brasil. Decreto no 50.744, de 8 de Junho de 1961. Cria o Parque Nacional de Sete Cidades, integrante da Seção de Parques e Florestas Nacionais do Serviço Florestal, do Ministério da Agricultura. Disponível em: <https://www2.camara.leg.br/legin/fed/ decret/1960-1969/decreto-50744-8-junho-1961-390270-publicacaooriginal-1-pe.html>. Acesso em: 28 ago. 2021.

Casas, A.; Lira, R.; Torres, I.; Delgado, A.; Moreno-Calles, A. I.; Rangel-Landa, S.; Blancas, J.; Larios, C.; Solís, L.; Pérez-Negrón, E.; Vallejo, M.; Parra, F.; Farfán-Heredia B.; Arellanes, Y.; Campos, N. Ethnobotany for sustainable ecosystem management: A regional perspective in the Tehuacán Valley. In: Lira, R.; Casas, A.; Blancas, J. (Eds.). Ethnobotany of Mexico: Interactions of people and plants in Mesoamerica. New York: Springer, 2016.

Cottam, G.; Curtis, J. T. The use of distance measures in phytosociological sampling. Ecology, v. 37, n. 3, p. 451-460, 1956. https://doi.org/10.2307/1930167

Cunha, L. V. F.; Albuquerque, U. P. Quantitative ethnobotany in an Atlantic Forest fragment of Northeastern Brazil: Implications to conservation. Environmental Monitoring and Assessment, v. 114, p. 1-25, 2006. https://doi.org/10.1007/s10661-006-1074-9

Feeny, P. Plant apparency and chemical defense. In: Wallace, J. W.; Nansel, R. L. (Eds.). Biological interactions between plants and insects: Recent advances in phytochemistry. Boston: Springer, 1976. v. 10.

Ferraz, J. S. F.; Albuquerque, U. P.; Meunier, I. M. J. Valor do uso e estrutura da vegetação lenhosa às margens do Riacho do Navio, Floresta, PE, Brasil. Acta Botanica Brasilica, v. 20, p. 125-134, 2006. https://doi.org/10.1590/S0102-33062006000100012

Hassan, I.; Shehu, A.; Zezi, A. U.; Magaji, M. G.; Ya'u, J. Ethnobotanical survey of medicinal plants commonly used in snakebites in North Western Nigeria. Journal of Medicinal Plants Research, v. 14, n. 9, p. 468-474, 2020. https://doi.org/10.5897/JMPR2020.6962

Galeano, G. Forest use at the Pacific Coast of Chocó, Colômbia: A quantitative approach. Economic Botany, v. 54, n. 3, p. 358-376, 2000. https://doi.org/10.1007/BF02864787

Gaoue, O. G.; Coe, M. A.; Bond, M.; Hart, G.; Seyler, B. C.; McMillen, H. Theories and major hypotheses in ethnobotany. Economic Botany, v. 71, n. 3, p. 269-287, 2017. https://doi.org/10.1007/s12231-017-9389-8

Guerra, N. M.; Carvalho, T. K. N.; Ribeiro, J. E. S.; Ribeiro, J. P. O.; Barbosa, A. R.; Limam, J. R. F.; Alves, C. A. B.; Oliveira, R. S.; Lucena, R. F. P. Ecological apparency hypothesis and plant utility in the Semiarid Region of Brazil. Ethnobotany Research and Applications, v. 14, p. 423-435, 2015.

Jiménez-Escobar, N. D.; Rangel-Ch, J. O. La abundancia, la dominancia y sus relaciones con el uso de la vegetación arbórea en la Bahía de Cispatá, Caribe Colombiano: Abundance, dominance and their relationship to use of tree species in Cispatá Bay, Caribbean Region of Colombia. Caldasia, v. 34, n. 2, p. 347-366, 2012.

La Torre-Cuadros, M. A.; Islebe, G. A. Traditional ecological knowledge and use of vegetation in Southeastern México: A case study from Solferino, Quintana Roo. Biodiversity and Conservation, v. 12, p.2455-2476, 2003. https://doi.org/10.1023/ A:1025861014392

Lawrence, A.; Phillips, O. L.; Ismodes, A. R.; Lopez, M.; Rose, S.; Wood, D.; Farfan, A. J. Local values for harvested forest plants in Madre de Dios, Peru: Towards a more contextualised interpretation of quantitative ethnobotanical data. Biodiversity and Conservation, v. 14, n, 1, p. 45-79, 2005. https://doi.org/10.1007/s10531-005-4050-8 
Lima, J. R. F.; Alves, C. A. B.; Ribeiro, J. E. S.; Cruz, D. D.; Mourão, J. S.; Torre Cuadros, M. L. A.; Lucena, R. F. P. Uso e disponibilidade de espécies vegetais nativas no semiárido do Nordeste do Brasil: uma análise da hipótese da aparência ecológica. REDE - Revista Eletrônica do PRODEMA, v. 10, n. 1, p. 110-131, 2016.

Lima-Nascimento, A. M.; Bento-Silva, J. S.; Lucena, C. M.; Lucena, R. F. P. Ethnobotany of native cacti in the Northeast Region of Brazil: Can traditional use influence availability? Acta Botanica Brasilica, v. 33, n. 2, p. 350-359, 2019. https://doi.org/10.1590/0102$33062019 a b b 0166$

Lucena, R. F. P.; Albuquerque, U. P.; Araújo, E. L. Does the local availability of woody Caatinga plants (Northeastern Brazil) explain their use value? Economic Botany, v. 61, n. 4, p. 347-361, 2007. https://doi.org/10.1663/0013-0001(2007)61[347:DTLAOW] 2.0.CO;2

Lucena, R. F. P.; Medeiros, P. M.; Araújo, E. L.; Alves, A. G. C.; Albuquerque, U. P. The ecological apparency hypothesis and the importance of useful plants: An assessment based on value-use. Journal of Environmental Management, v. 96, p. 106-115, 2012a. https://doi.org/10.1016/j.jenvman.2011.09.001

Lucena, R. F. P.; Leite, A. P.; Pedrosa, K. M.; Lucena, C. M.; Vasconcelos Neto, C. F. A.; Ribeiro, J. P. O. O uso de espécies vegetais no Vale do Piancó pode ser explicado por sua disponibilidade local? Revista de Biologia e Farmácia, v. esp., 2012b.

Lucena, R. F. P.; Lucena, C. M.; Araújo, E. L.; Alves, A. G. C.; Albuquerque, U. P. Conservation priorities of useful plants from different techniques of collection and analysis of ethnobotanical data. Anais da Academia Brasileira de Ciências, v. 85, n. 1, p. 169-186, 2013. https://doi.org/10.1590/S0001-37652013005000013

Lucena, R. F. P.; Sousa, R. F.; Guerra, N. M.; Ribeiro, J. E. S.; Leite, A. P.; Abreu, D. B. O.; Carvalho, T. K. N.; Trovão, D. M. B. M.; Alves, C. A. B.; Alves, R. R. N.; Borges, P. F.; Andrade, L. A.; Souto, J. S.; Sousa Júnior, S. P.; Nunes, E. N. The ecological apparency hypothesis and dry tropical forests: An ethnobotanical assessment. Etnoecológica, v. 10, n. 9, p. 70-86, 2014.

Nunes, G. M.; Souza, A. S.; Ferreira, E. C.; Nunes, M. M.; Lins Filho, J. A.; Lucena, C. M.; Lucena, R. F. P. Estabelecendo prioridade de conservação para plantas medicinais no Estado da Paraíba, Nordeste do Brasil. Revista Brasileira de Gestão Ambiental e Sustentabilidade, v. 8, n. 19, p. 1029-1045, 2021. https://doi.org/10.21438/rbgas(2021) 081927

Murad, W.; Azizullah, A.; Adnan, M.; Tariq, A.; Khan, K. U.; Waheed, S.; Ahmad, A. Ethnobotanical assessment of plant resources of Banda Daud Shah, District Karak, Pakistan. Journal of Ethnobiology and Ethnomedicine, v. 9, n. 77, 2013. https://doi.org/10.1186/1746-4269-9-77

Mutchnick, P. A.; McCarthy, B. C. An ethnobotanical analysis of the tree species common to the subtropical moist forests of the Petén, Guatemala. Economic Botany, v. 51, n. 2, p. 158-183. 1997. https://doi.org/10.1007/BF02893110

Oliveira, T. C. S.; Sousa, V. F. S.; Silva, V. F.; Farias, R. R. S.; Andrade, I. M.; Castro, A. A. J. F. Structure and use of a rocky Cerrado in Northeastern Brazil: Does the Ecological Appearancy Hypothesis explain this relationship? Journal of Experimental Agriculture International, v. 38, n. 5, p. 1-10, 2019. https://doi.org/10.9734/jeai/2019/v38i530312 
Oliveira, M. E. A.; Castro, A. A. J. F.; Martins, F. R. Fisionomias vegetacionais do Parque Nacional de Sete Cidades, Piauí, NE do Brasil. In: Araújo, A. S. F. (Ed.). Biodiversidade de solo e plantas (Parque Nacional de Sete Cidades). Teresina: EDUFPI, 2017. p. 12-34.

Paz y Minõ, G.; Balslev, H.; Valencia, R.; Mena, P. Lianas utilizadas por los indígenas Siona-Secoya de la Amazonía del Ecuador. Quito: Ecociencia, 1991. (Reportes Técnicos, 1).

Phillips, O.; Gentry, A. H. The useful plants of Tambopata, Peru: I. Statistical hypotheses tests with a new quantitative technique. Economic Botany, v. 47, n. 1, p. 15-32, 1993a. https://doi.org/10.1007/BF02862203

Phillips, O.; Gentry, A. H. The useful plants of Tambopata, Peru: II. Additional hypothesis testing in quantitative ethnobotany. Economic Botany, v. 47, n. 1, p.33-43, 1993b. https://doi.org/10.1007/BF02862204

Pinho Júnior, G. V.; Guido, L. D. F. E.; Nascimento, A. R. T. Relações entre Valor de Uso e parâmetros fitossociológicas em duas fitofisionomias de Cerrado no Município de Uberlândia, MG. Bioscience, v. 29, n. 5, p. 1339-1349, 2013.

Rhoades, D. F.; Cates, R. G. Toward a general theory of plant antiherbivore chemistry. In: Wallace, J. W.; Nansel, R. L. (Eds.). Biological interactions between plants and insects: Recent advances in phytochemistry, Boston: Springer, 1976. v. 10.

Ribeiro, J. E. S.; Carvalho, T. K. N.; Ribeiro, J. P. O.; Guerra, N. M.; Silva, N.; Pedrosa, K. M.; Alves, C. A. B.; Sousa J\&uacute;nior, S. P.; Souto, J. S; Nunes, A. T.; Lima, J. R. F.; Oliveira, R. S.; Lucena, R. F. P. Ecological apparency hypothesis and availability of useful plants: Testing different use values. Ethnobotany Research and Applications, v. 12, p. 415-432, 2014a. https://doi.org/10.17348/era.12.0.415-432

Ribeiro, J. P. O.; Carvalho, T. K. N.; Ribeiro, J. E. S.; Sousa, R. F.; Lima, J. R. F.; Oliveira, R. S.; Alves, C. A. B.; Jardim, J. G.; Lucena, R. F. P. Can ecological apparency explain the use of plant species in the Semi-Arid Depression of Northeastern Brazil? Acta Botanica Brasilica, v. $28, \quad$ n. 3, p. 476-483, 2014b. https://doi.org/10.1590/0102$33062014 \mathrm{abb} 2758$

Rodríguez M. A.; Angueyra, A.; Cleef, A. M.; Van Andel, T. Ethnobotany of the Sierra Nevada del Cocuy-Güicán: Climate change and conservation strategies in the Colombian Andes. Journal of Ethnobiology and Ethnomedicine, v. 14, n. 34, 2018. https://doi.org/10.1186/s13002-018-0227-6

Rossato, S. C.; Leitão-Filho, H. F.; Begossi, A. Ethnobotany of caiçaras of the Atlantic Forest

$\begin{array}{llllll}\text { Coast (Brazil). Economic Botany, v. 53, n. 4, p. 387-395, } 1999 . & \text {. }\end{array}$ https://doi.org/10.1007/BF02866716

Sher, H.; Elyemeni, M.; Sher, H.; Hussain, K. Ethnobotanical and economic observations of some plant resources from the Northern parts of Pakistan. Ethnobotany Research and Applications, v. 9, p. 27-41, 2011. https://doi.org/10.17348/era.9.0.27-41

Singh A. G.; Kumar A.; Tewari D. D. An ethnobotanical survey of medicinal plants used in Terai Forest of Western Nepal. Journal Ethnobiology and Ethnomedicine, v. 8, n. 19, p. 2012. https://doi.org/10.1186/1746-4269-8-19

Sokal, R. R.; Rholf, F. G. Biometry. New York: Freeman and Company, 1995.

Sop, T. K.; Oldeland, J.; Bognounou, F.; Schmiedel, U.; Thiombiano, A. Ethnobotanical knowledge and valuation of woody plants species: A comparative analysis of three ethnic groups from the Sub-Sahel of Burkina Faso. Environment, Development and Sustainability, v. 14, p. 627-649, 2012. https://doi.org/10.1007/s10668-012-9345-9 
Souza, A. P.; Costa, F. C. P.; Alencar, R. F.; Lima, S. F. B. Exploração e utilização do potencial madeireiro da Caatinga no Município de Aurora - Estado do Ceará. Pesquisa e Ensino em Ciências Exatas e da Natureza, v. 2, n. 2, 2018.

Stagegaard, J.; Sorensen, M.; Kvist. E. L. P. Estimations of the importance of plant resources extracted by inhabitants of the Peruvian Amazon flood plains. Perspectives in Plant $\begin{array}{lllll}\text { Ecology. Evolution and Systematics, } & \text { v. 5, } & \text { n. 2, p. 103-122, } 2002 .\end{array}$ https://doi.org/10.1078/1433-8319-00026

Thomas, E.; Vandebroek, I.; Van Damme, P. Valuation of forest and plant species in indigenous territory and National Park Isiboro-Sécure, Bolivia. Economic Botany, v. 63, n. 3, p. 229-241, 2009. https://doi.org/10.1007/s12231-009-9084-5

Trindade, M. R. O.; Jardim, J. G.; Casas, A.; Guerra, N. M.; Lucena, R. F. P. Availability and use of woody plant resources in two areas of Caatinga in Northeastern Brazil. Ethnobotany Research and Applications, v. 14, p. 313-330, 2015. https://doi.org/10.17348/ era.14.0.313-330

Tunholi, V. P.; Ramos, M. A.; Scariot, A. Availability and use of woody plants in an agrarian reform settlement in the Cerrado of the State of Goiás, Brazil. Acta Botanica Brasilica, v. 27, n. 3, p. 604-612, 2013. https://doi.org/10.1590/S0102-33062013000300018

Uprety, Y.; Poudel, R. C.; Shrestha, K. K.; Rajbhandary, S.; Tiwari, N. N.; Shrestha, U. B.; Asselin, H. Diversity of use and local knowledge of wild edible plant resources in Nepal. Journal of Ethnobiology and Ethnomedicine, v. 8, n. 16, 2012. https://doi.org/10.1186/ 1746-4269-8-16

Informação da Licença: Este é um artigo Open Access distribuído sob os termos da Licença Creative Commons Attribution, que permite uso irrestrito, distribuição e reprodução em qualquer meio, desde que a obra original seja devidamente citada. 